\title{
Graviton production in the scaling of a long-cosmic-string network
}

\author{
Kostas Kleidis, ${ }^{1}$ Apostolos Kuiroukidis, ${ }^{2}$ Demetrios B. Papadopoulos, ${ }^{2}$ and Enric Verdaguer ${ }^{3}$ \\ ${ }^{1}$ Department of Mechanical Engineering, Technological Education Institute of Serres, GR-62124 Serres, Greece \\ ${ }^{2}$ Department of Physics, Aristotle University of Thessaloniki, GR-54124 Thessaloniki, Greece \\ ${ }^{3}$ Departament de Fisica Fonamental and Institut de Ciences del Cosmos, Universitat de Barcelona, \\ Avinguda Diagonal 647, E-08028 Barcelona, Spain
}

(Received 26 September 2011; published 23 December 2011)

In a previous paper [K. Kleidis, D. B. Papadopoulos, E. Verdaguer, and L. Vlahos, Phys. Rev. D 78, 024027 (2008).] we considered the possibility that (within the early-radiation epoch) there has been (also) a short period of a significant presence of cosmic strings. During this radiation-plus-strings stage the Universe matter-energy content can be modeled by a two-component fluid, consisting of radiation (dominant) and a cosmic-string fluid (subdominant). It was found that, during this stage, the cosmological gravitational waves - that had been produced in an earlier (inflationary) epoch - with comoving wave numbers below a critical value (which depends on the physics of the cosmic-string network) were filtered, leading to a distorsion in the expected (scale-invariant) cosmological gravitational wave power spectrum. In any case, the cosmological evolution gradually results in the scaling of any long-cosmic-string network and, hence, after a short time interval, the Universe enters into the late-radiation era. However, along the transition from an early-radiation epoch to the late-radiation era through the radiation-plus-strings stage, the time dependence of the cosmological scale factor is modified, something that leads to a discontinuous change of the corresponding scalar curvature, which, in turn, triggers the quantum-mechanical creation of gravitons. In this paper we discuss several aspects of such a process, and, in particular, the observational consequences on the expected gravitational-wave power spectrum.

DOI: $10.1103 /$ PhysRevD.84.124044

PACS numbers: 04.30.-w, 11.25.-w, 98.80.Cq

\section{INTRODUCTION}

Cosmological gravitational waves (CGWs) represent small-scale perturbations to the Universe metric tensor [1]. Since gravity is the weakest of the four known forces, these metric corrections decouple from the rest of the Universe at very early times, presumably at the Planck epoch [2]. Their subsequent propagation is governed by the space-time curvature [3], encapsulating in the field equations the inherent coupling between relic gravitational waves (GWs) and the Universe matter content; the latter being responsible for the background gravitational field [4].

In this context, we explore the creation of primordial GWs during the scaling of a long-cosmic-string network (see, e.g., [5]), i.e., in the transition from a cosmological model in which the matter content can be modeled by a two-component fluid - consisting of radiation (dominant) and cosmic strings (subdominant) - to a pure radiationdominated Universe.

Cosmic strings are one-dimensional objects that can be formed as linear defects at a symmetry-breaking phase transition [6,7]. If they exist, they may help us to explain some of the large-scale structures seen in the Universe today, such as the gravitational lenses [8]. They may also serve as seeds for density perturbations [9,10], as well as potential sources of relic gravitational radiation $[11,12]$.

The presence of cosmic strings in a radiation model is responsible for the constancy of the effective potential which drives the temporal evolution of a CGW, leading to a critical value $\left(k_{c}\right)$ of the comoving wave number, which discriminates between the metric perturbations into oscillating $\left(k>k_{c}\right)$ and nonoscillating $\left(k<k_{c}\right)$ modes [13]. As a consequence, the propagation of CGWs through a radiation-plus-strings stage would leave imprints on their power spectrum. However, such a stage does not last very long, since, gradually, the production of loops smaller than the horizon results in the scaling of the long-cosmic-string network. According to this process, the linear defects form a self-similar configuration, the density of which, eventually, behaves as $R^{-4}$ and the Universe (re)enters in the pure-radiation era (see, e.g., [6]).

In the present article, we study the quantum-mechanical production of gravitons in the transition of the Universe from an early-radiation epoch to the late-radiation era through the radiation-plus-strings stage. Several theoretical (and observational) consequences are discussed.

The paper is organized as follows: In Sec. II, we summarize the theory of CGWs propagating in curved spacetime, including the implications arising from the constancy of the effective potential. In Sec. III, we take advantage of the scaling of a long-cosmic-string network to arrive at the late-radiation era. In Sec. IV, we demonstrate that, due to the modification in the time-dependence of the cosmological scale factor along this transition, the scalar curvature changes discontinuously; hence, gravitons are created, and, in Sec. V, we explore the corresponding power spectrum. The high-frequency part $\left(k>k_{c}\right)$ of this spectrum acquires a characteristic profile, resulting in a periodic function of 
the frequency. Finally, in Sec. VI, we evaluate the (integrated) energy density of the CGWs created in the scaling of the long-cosmic-string network and we compare it with the corresponding quantity predicted by inflation (see, e.g., [11]). It is worth noting that the energy density of the CGWs created in the transition of the Universe from an early-radiation epoch to the late-radiation era through a radiation-plus-strings stage, not only is added to the corresponding inflationary quantity. In fact, at times $10^{-19} \mathrm{sec} \leq t \leq 6540 \mathrm{sec}$, i.e., within the first (and, certainly, most interesting) $109 \mathrm{~min}$ of the universal evolution, it dominates over every other form of GW-energy density.

As regards the space-time geometry itself, we confine ourselves to the spatially flat Friedmann-RobertsonWalker (FRW) model. This model appears to interpret adequately both the observational data related to the known thermal history of the Universe and the theoretical approach to cosmic-string configurations [6]. Therefore, it seems to be the most appropriate candidate for the curved background needed for this study.

\section{CGWS IN A FRIEDMANN MODEL}

The quantum-gravitational creation of gravitons in an expanding FRW Universe was first demonstrated by Grishchuk [14]. He showed that, in the linear approximation, the behavior of a CGW propagating in curved spacetime is identical to that of a massless, minimally-coupled scalar field. In particular, each of the two polarization states of the metric perturbation satisfy the Klein-Gordon equation

$$
h_{i j ; \alpha}^{; \alpha}=0,
$$

where Greek indices refer to the four-dimensional spacetime, Latin indices refer to the corresponding threedimensional spatial slices and the semicolon denotes covariant derivative. The quantization of primordial GWs and that of the minimally-coupled, massless scalar fields also proceeds along identical lines, as it was demonstrated by Ford and Parker $[15,16]$.

A weak CGW $\left(\left|h_{i j}\right| \ll 1\right)$ propagating in a spatially flat FRW cosmological model is defined as [11]

$$
d s^{2}=c^{2} d t^{2}-R^{2}(t)\left(\delta_{i j}+h_{i j}\right) d x^{i} d x^{j},
$$

where $c$ is the velocity of light, $\delta_{i j}$ is the Kronecker symbol, and the dimensionless scale factor $R(t)$ is a solution to the Friedmann equations with matter content in the form of a perfect fluid. In terms of the conformal time,

$$
\tau=\int \frac{d t}{R(t)},
$$

the solution to the Klein-Gordon Eq. (1) is a linear superposition of plane-wave modes

$$
h_{i j}\left(\tau, x^{r}\right)=\phi_{k}(\tau) \varepsilon_{i j} e^{\imath k_{r} x^{r}},
$$

where $\phi_{k}(\tau)$ is a (complex) function of time and $\varepsilon_{i j}$ is the polarization tensor, depending only on the direction of the comoving wave-vector $k_{r}$. For a fixed wave number $k^{2}=$ $\sum k_{r}^{2}$, the time-dependent part of the corresponding GW mode satisfies the second-order differential equation (see, e.g., [17])

$$
\phi_{k}^{\prime \prime}+2 \frac{R^{\prime}}{R} \phi_{k}^{\prime}+k^{2} c^{2} \phi_{k}=0,
$$

where a prime denotes differentiation with respect to $\tau$. Under the further decomposition

$$
h_{k}(\tau)=\phi_{k}(\tau) R(\tau) .
$$

Equation (5), which governs the temporal evolution of a CGW in a FRW Universe, is written in the form

$$
h_{k}^{\prime \prime}+\left(k^{2} c^{2}-\frac{R^{\prime \prime}}{R}\right) h_{k}=0 .
$$

Equation (7) can be treated as the Schrödinger equation, for a particle moving in the presence of the effective potential

$$
V_{\mathrm{eff}}=\frac{R^{\prime \prime}}{R} .
$$

To solve this equation, one needs an evolution formula for the cosmological model under consideration.

In terms of the conformal time, the spatially flat FRW model is a solution to the Friedmann equation

$$
\left(\frac{R^{\prime}}{R^{2}}\right)^{2}=\frac{8 \pi G}{3} \rho(\tau)
$$

(where $G$ is Newton's universal constant of gravitation), with matter content in the form of a perfect fluid source, $T_{\mu \nu}=\operatorname{diag}\left(\rho c^{2},-p,-p,-p\right)$, which obeys the conservation law

$$
\rho^{\prime}+3 \frac{R^{\prime}}{R}\left(\rho+\frac{1}{c^{2}} p\right)=0
$$

and the equation of state

$$
p=\left(\frac{m}{3}-1\right) \rho c^{2},
$$

where $\rho(\tau)$ and $p(\tau)$ represent the mass density and the pressure, respectively.

The linear equation of state (11) covers most of the matter components considered to drive the evolution of the Universe, such as quantum vacuum $(m=0)$, a network of domain walls $(m=1)$, a gas of cosmic strings $(m=2)$, dust $(m=3)$, radiation $(m=4)$, and Zel'dovich ultrastiff matter $(m=6)$. For each component, the continuity Eq. (10) yields

$$
\rho=\frac{M_{m}}{R^{m}},
$$


where $M_{m}$ is an integration constant. Provided that the various components do not interact with each other, a mixture of them obeys [18]

$$
\rho=\sum_{m} \frac{M_{m}}{R^{m}}
$$

where, now, Eq. (10) holds for every matter constituent separately.

\section{A. Constancy of the effective potential}

There is a case of particular interest, involved in the temporal evolution of a primordial CGW, in which the effective potential is constant for every $\tau$, namely

$$
\frac{R^{\prime \prime}}{R}=\text { const }=\frac{8 \pi G}{3} M_{2},
$$

where $M_{2}$ is a non-negative constant of units $\mathrm{gr} / \mathrm{cm}^{3}$ [13]. In this case, Eq. (7) is written in the form

$$
h_{k}^{\prime \prime}+\left(k^{2} c^{2}-\frac{8 \pi G}{3} M_{2}\right) h_{k}=0 .
$$

From Eq. (15), it becomes evident that, as long as $M_{2} \neq 0$, there exists a critical value of the comoving wave number,

$$
k_{c}=\sqrt{\frac{8 \pi G}{3 c^{2}} M_{2}},
$$

which discriminates between the primordial GWs into oscillating $\left(k>k_{c}\right)$ and nonoscillating $\left(k<k_{c}\right)$ modes. According to [13], such a property is met in a cosmological model with matter content in the form of a two-component fluid with

$$
\rho(\tau)=\frac{M_{2}}{R^{2}}+\frac{M_{4}}{R^{4}},
$$

where $M_{4}$ is an integration constant, also of units $\mathrm{gr} / \mathrm{cm}^{3}$.

For $M_{4}=0$, Eq. (17) results in $\rho \sim R^{-2}$, which, by virtue of Eqs. (11) and (12), leads to $p=-\frac{1}{3} \rho c^{2}$. This case, now known as the string-dominated Universe [19], was considered by Ford and Parker [15], in their effort for a natural renormalization of the energy-momentum tensor of the gravitational-perturbation field.

A string-dominated Universe does not seem likely and, therefore, in this article, we consider $M_{4} \neq 0$. Accordingly, upon consideration of Eqs. (12) and (13), we distinguish two cases, in Eq. (17), with respect to $M_{2}$ : (i) $M_{2}=0$ and (ii) $M_{2} \neq 0$. We consider each one of them separately.

\section{B. CGWs in a radiation-dominated Universe}

For $M_{2}=0$, Eq. (17) reads

$$
\rho(\tau)=\frac{M_{4}}{R^{4}} .
$$

In view of Eq. (12), this is the matter-energy content of a Universe filled with relativistic particles_radiation. Now, the effective potential vanishes and Eq. (15) is written in the form

$$
h_{k}^{\prime \prime}+k^{2} c^{2} h_{k}=0,
$$

i.e., it is a simple harmonic oscillator equation; like a wave propagating in flat space-time, where there is no particle production [20]. Therefore, the choice of a solution to Eq. (19) corresponds to the choice of an initial (or final) quantum state for the gravitational-perturbation field. Equation (19) admits the solution

$$
h_{k}^{\text {in }}(\tau)=\sqrt{\frac{2}{\pi k c}} e^{-\imath k c \tau}
$$

and the gravitational-perturbation field is in the welldefined adiabatic-vacuum state [21].

\section{CGWs in the presence of cosmic strings}

Setting, in Eq. (17), $M_{2} \neq 0$, corresponds to a radiation model contaminated by cosmic strings, in which, the two constituents (relativistic particles and linear defects) do not interact with each other [cf. Equation (13)]. In this case, the constant effective potential (14), is related to the initial amount of the linear defects in the "mixture," $M_{2}$. Now, Eq. (15) results in

$$
h_{k}^{\prime \prime}+\left(k^{2}-k_{c}^{2}\right) c^{2} h_{k}=0 .
$$

For every $0<k<\infty$, the general solution to Eq. (21) can be expressed in the form

$$
h_{k}(\tau)=\sqrt{\frac{2}{\pi \gamma k c}}\left[c_{1} e^{-l \gamma k c \tau}+c_{2} e^{+l \gamma k c \tau}\right]
$$

where $c_{1}$ and $c_{2}$ are complex numbers, satisfying the Wronskian condition

$$
\left|c_{1}\right|^{2}-\left|c_{2}\right|^{2}=1
$$

(as a consequence of the mode normalization when the gravitational perturbations are quantized), and we have introduced the parameter

$$
\gamma=\left[1-\left(\frac{k_{c}}{k}\right)^{2}\right]^{1 / 2},
$$

which measures the departure from the pure-radiation case, given by Eq. (20).

\section{A TALE OF COSMIC STRINGS}

The presence of cosmic strings in the Universe is purely a question of topology [22]. In particular, after inflation (and reheating) the Universe enters an early-radiation (ER) epoch [23], during which the temperature drops monotonically $\left(T \sim R^{-1}\right)$. This cooling process may have resulted in the breaking of a fundamental U(1) local gauge symmetry, which, in turn, led to the formation of linear topological 
defects (for a detailed analysis see [6] and/or [7]). Within this era, the cosmological scale factor behaves as

$$
R_{\mathrm{ER}}(t)=\left(\frac{t}{t_{\mathrm{cr}}}\right)^{1 / 2} \Rightarrow R_{\mathrm{ER}}(\tau)=\frac{\tau}{\tau_{\mathrm{cr}}},
$$

where $\tau_{\text {cr }}=2 t_{\text {cr }}$ marks the (conformal) time at which the cosmic strings are formed, and we have normalized $R\left(t_{\mathrm{cr}}\right)$ to unity.

By the time these linear defects are formed, they are moving in a very dense environment and, hence, their motion is heavily damped, due to string-particle scattering [24-26]. This friction becomes subdominant to expansion damping [24] at

$$
t_{*}=\left(\frac{G \mu}{c^{2}}\right)^{-1} t_{\mathrm{cr}} \Rightarrow \tau_{*}=\left(\frac{G \mu}{c^{2}}\right)^{-1 / 2} \tau_{\mathrm{cr}},
$$

where $\mu$ is the mass per unit-length of the linear defect. For $\tau \geq \tau_{*}$, the motion of the long cosmic strings is essentially independent of anything else in the Universe and soon they acquire relativistic velocities [6]. Now, the gas of cosmic strings does not interact with the fluid of relativistic particles and, hence, Eq. (17) holds. Therefore, we may consider that, for $\tau \geq \tau_{*}$, the evolution of the curved space-time is driven by a two-component fluid, consisting of radiation (dominant) and cosmic strings (subdominant). Accordingly, $\tau_{*}$ marks the beginning of a radiation-plusstrings stage, during which, the Friedmann Eq. (9) admits the solution [13]

$$
R(\tau)=\sqrt{\frac{M_{4}}{M_{2}}} \sinh \sqrt{\frac{8 \pi G}{3} M_{2}} \tau .
$$

Nevertheless, the scale factor (27) can drive the Universe expansion only for a short period of time after $\tau_{*}$, since cosmic strings should (at any time) be a small proportion of the Universe matter-energy content. This means that the equation of state (17) has validity only for a limited time period, otherwise, cosmic strings would, eventually, dominate the Universe energy density (see, e.g., [19]).

In fact, the radiation-plus-strings stage (if it ever existed) does not last very long. Numerical simulations in the early 90's [27-30], as well as their present-time counterparts [31-33], suggest that, after the friction becomes unimportant, the production of loops smaller than the horizon, gradually results in the scaling of the long-cosmic-string network. According to this process, the linear defects form a self-similar configuration, the density of which, eventually, behaves as $R^{-4}$. In this way, at some (physical) time $t_{\mathrm{sc}}>t_{*}$ the Universe reenters in the (late) radiation (LR) era,

$$
R_{\mathrm{LR}}(t)=R_{\mathrm{sc}}\left(\frac{t}{t_{\mathrm{sc}}}\right)^{1 / 2} \Rightarrow R_{\mathrm{LR}}(\tau)=R_{\mathrm{sc}} \frac{\tau}{\tau_{\mathrm{sc}}},
$$

before it can become string-dominated. The duration of the radiation-plus-strings stage is quite uncertain, mostly due to the fact that numerical simulations (which revealed the scaling of the long-strings network) can be run for relatively limited times. For example, the earliest treatments $[29,30]$ suggested that $t_{\mathrm{sc}} \simeq 30 t_{*}\left(\tau_{\mathrm{sc}} \simeq 5.5 \tau_{*}\right)$, while, the most recent ones [32] raise this value to $t_{\mathrm{sc}} \simeq 300 t_{*}\left(\tau_{\mathrm{sc}} \simeq\right.$ $17 \tau_{*}$ ). According to Eq. (40) of [13], the former result leads to $k_{c} c \Delta \tau=k_{c} c\left(\tau_{\mathrm{sc}}-\tau_{*}\right)=0.09$, while, adoption of the latter result, would lead to $k_{c} c \Delta \tau=0.32$.

\section{GRAVITON CREATION}

Around $\tau_{*}$ and $\tau_{\mathrm{sc}}$, both $R(\tau)$ and $R^{\prime}(\tau)$ can be matched to be continuous (cf. [13]), but $R^{\prime \prime}(\tau)$ is essentially discontinuous, acquiring a nonzero value through Eq. (14). Consequently, the scalar curvature of the space-time changes discontinuously both at $\tau_{*}$ and at $\tau_{\mathrm{sc}}$. A discontinuous change in the scalar curvature produces gravitons [20,34].

As a consequence, the evolution of the CGW modes in the transition of the Universe from an early-radiation epoch to the late-radiation era through a radiation-plus-strings stage, is modified as follows:

(i) For $\tau \leq \tau_{*}$ the effective potential (14) vanishes. The temporal evolution of the metric perturbations is governed by Eq. (19), admitting the solution (20) and the gravitational-perturbation field is in an adiabatic-vacuum state.

(ii) For $\tau_{*}<\tau<\tau_{\mathrm{sc}}$ the effective potential reduces to a nonvanishing constant $\left(V_{\text {eff }}=k_{c}^{2} c^{2}\right)$ and the evolution of the metric perturbations is driven by Eq. (21). Because of the discontinuous change of the scalar curvature at $\tau_{*}$, during the radiation-plusstrings stage the gravitational-perturbation field is no longer in its vacuum state and the general solution to Eq. (21) is the linear superposition of positive- and negative-frequency solutions (22). Moreover, for every $\tau_{*}<\tau<\tau_{\mathrm{sc}}$, the primordial CGWs, being discriminated by the value of their comoving wave number (in comparison to $k_{c}$ ), do not evolve in a unique way: The GW modes with $k>k_{c}$ perform oscillations within the horizon, while those of $k<k_{c}$ remain outside of the Hubble sphere and grow exponentially [13].

(iii) Finally, for $\tau \geq \tau_{\mathrm{sc}}, V_{\mathrm{eff}}=0$ and the temporal evolution of a metric perturbation in curved space-time is (once again) governed by Eq. (19). However, due to the discontinuity of the scalar curvature at $\tau_{\mathrm{sc}}$, this time, the general solution to Eq. (19) is written in the form

$$
h_{k}^{\text {out }}(\tau)=\sqrt{\frac{2}{\pi k c}}\left(\alpha_{k} e^{-\imath k c \tau}+\beta_{k} e^{+l k c \tau}\right),
$$

where the (Bogolubov) coefficients $\alpha_{k}$ and $\beta_{k}$ should satisfy the Wronskian condition 


$$
\left|\alpha_{k}\right|^{2}-\left|\beta_{k}\right|^{2}=1 \text {. }
$$

Equation (29) determines the final quantum state of the gravitational-perturbation field. As a result of conformal invariance, this state is also an adiabatic vacuum, but, as long as $\beta_{k} \neq 0$, it differs from the corresponding state of the early-radiation epoch. Clearly, the occurrence of a nonzero $\beta_{k}$ in the late-radiation era, would signal that $h_{k}^{\text {out }}(\tau)$ is not a pure positive-frequency solution, but contains also a negative-frequency component. This means that, if the in-state of the gravitational-perturbation field is taken to be vacuum, then, particles are found in the outstate [21].

In what follows, we discuss the evolution of the modes (20), both with $k>k_{c}$ and with $k<k_{c}$, in the transition of the Universe from the early-radiation epoch to the lateradiation era, through a radiation-plus-strings stage. The coefficients $c_{1}, c_{2}, \alpha_{k}$ and $\beta_{k}$ can be determined by the requirement that $h_{k}(\tau)$ and its first derivative $h_{k}^{\prime}(\tau)$ are continuous across the boundaries $\tau_{*}$ and $\tau_{\mathrm{sc}}$.

Matching the modes (20) and (22), as well as their first derivatives, at $\tau=\tau_{*}$, we obtain

$$
c_{1}=\frac{\gamma+1}{2 \sqrt{\gamma}} e^{\imath k c(\gamma-1) \tau_{*}}
$$

and

$$
c_{2}=\frac{\gamma-1}{2 \sqrt{\gamma}} e^{-l k c(\gamma+1) \tau_{*}},
$$

for which the Wronskian condition (23) holds. Similarly, matching the modes (22) and (29), as well as their first derivatives, at $\tau=\tau_{\mathrm{sc}}$, we obtain

$$
\alpha_{k}=\frac{1}{4 \gamma}\left[(\gamma+1)^{2} e^{-l(\gamma-1) k c \Delta \tau}-(\gamma-1)^{2} e^{+l(\gamma+1) k c \Delta \tau}\right]
$$

and

$$
\beta_{k}=\imath \frac{\gamma^{2}-1}{2 \gamma} \sin (\gamma k c \Delta \tau) e^{-\imath k c\left(\tau_{*}+\tau_{\mathrm{sc}}\right)},
$$

where $\Delta \tau=\tau_{\mathrm{sc}}-\tau_{*}$ and we have taken into account Eqs. (31) and (32). By virtue of Eq. (34), the number of gravitons [21] created in the mode denoted by $0<k<\infty$, is written in the form

$$
N_{k}=\left|\beta_{k}\right|^{2}=\frac{1}{4 \gamma^{2}}\left(\gamma^{2}-1\right)^{2} \sin ^{2}(\gamma k c \Delta \tau)
$$

and the normalization condition, $\left|\alpha_{k}\right|^{2}-\left|\beta_{k}\right|^{2}=1$, holds. The observable quantity $N_{k}$ possesses a series of very interesting properties, depending on the value of $k$ in comparison to $k_{c}$. We distinguish the following cases:

\section{A. Oscillating modes}

In this case, $k>k_{c}$ and Eq. (35) yields

$$
N_{k>k_{c}}=\frac{k_{c}^{4}}{4 k^{2}} \frac{\sin ^{2}\left(c \Delta \tau \sqrt{k^{2}-k_{c}^{2}}\right)}{k^{2}-k_{c}^{2}} .
$$

Obviously, for $k_{c}=0, N_{k>k_{c}}$ vanishes, i.e., no gravitons are created in the absence of cosmic strings.

On the other hand, for $k \rightarrow k_{c}$ and/or $\Delta \tau \rightarrow 0$, Eq. (36) results in

$$
N_{k>k_{c}} \rightarrow \frac{k_{c}^{4}}{4 k^{2}} c^{2} \Delta \tau^{2} .
$$

Now, we distinguish the following cases:

(a) $\Delta \tau=0$ : In this case, $N_{k>k_{c}}=0$. Although this may look like an unexpected result, it is not. Usually, the instantaneous transition from one epoch to another may lead to pathological results, especially when high-frequency modes are concerned (see, e.g., [21]). However, in our case, as long as $\Delta \tau=0$ no transition ever takes place. The Universe remains in the (early) radiation era, where no gravitons are produced.

(b) $k=k_{c}$ : In this case, the number of gravitons created in the scaling of a long-cosmic-string network acquires the constant value

$$
N_{k_{c}}=\frac{k_{c}^{2}}{4} c^{2} \Delta \tau^{2} .
$$

Equation (38) suggests that, the number of gravitons in the ground state $\left(k_{c}\right)$ of the oscillating modes is well-defined and finite.

(c) Finally, for $k \gg k_{c}$ and $k \rightarrow \infty, N_{k>k_{c}} \rightarrow 0$ and particle production ceases. Consequently, there should be no ultraviolet divergences.

On the other hand, according to Eq. (36), the expectation value of the number operator, $N_{k>k_{c}}$, varies periodically with $k$. Hence:

(i) For comoving wave numbers satisfying the condition

$$
c \Delta \tau \sqrt{k^{2}-k_{c}^{2}}=m \pi(m=1,2, \ldots),
$$

$N_{k>k_{c}}=0$, admitting its absolute minimum value. The case $m=0$ is excluded due to the fact that $N_{k_{c}} \neq 0$. As a consequence, creation of gravitons with comoving wave numbers

$$
k_{m}^{2}=k_{c}^{2}+m^{2} \frac{\pi^{2}}{(c \Delta \tau)^{2}}(m=1,2, \ldots),
$$

never occurs by this mechanism, a case which (in quantum mechanics) is known as perfect transmission (see, e.g., [35], pp. 101-104). Clearly, the spectrum of these states is discrete and their position on 
the $k$-axis is determined (solely) by the choice of $\Delta \tau \neq 0$.

(ii) On the other hand, the values of $k>k_{c}$ that label the maxima of $N_{k>k_{c}}$ obey the equation

$$
\left(c \Delta \tau \sqrt{k^{2}-k_{c}^{2}}\right) \cot \left(c \Delta \tau \sqrt{k^{2}-k_{c}^{2}}\right)=2-\left(\frac{k_{c}}{k}\right)^{2},
$$

which can be solved numerically, to give

$$
k_{n}^{2} \simeq k_{c}^{2}+\left(n+\frac{49}{36}\right)^{2} \frac{\pi^{2}}{(c \Delta \tau)^{2}}(n=0,1,2, \ldots) .
$$

Once again, the position of these (discrete) states on the $k$-axis is determined (solely) by the choice of $\Delta \tau \neq 0$. Therefore, a possible detection of these waves, among other things, could reveal valuable information on the duration of the radiation-plusstrings stage, as well.

\section{B. Nonoscillating modes}

According to [13], for $\tau_{*}<\tau<\tau_{\text {sc }}$, the CGW modes with $k<k_{c}$ remain outside the horizon, where they grow exponentially. Nevertheless, from the quantum-mechanical point of view, low-frequency (nonoscillatory) modes are also created along the transition of the Universe through the radiation-plus-strings stage, in the form of waves that tunnel through the barrier $V_{\text {eff }}$ of width $\Delta \tau$. Of course, doing so, they are subject to an additional exponential decrease (see, e.g., [21]), which, eventually, may counterbalance their original growth. Nevertheless, due to the tunneling effect, they can also contribute to the CGWs with $k>k_{c}$ created during the radiation-plus-strings stage, and, therefore, they should be taken into account in the late-radiation era.

For $k<k_{c}$, the expectation value (35) of the number operator, $N_{k<k_{c}}=\left|\beta_{k<k_{c}}\right|^{2}$, is given by

$$
N_{k<k_{c}}=\frac{k_{c}^{4}}{4 k^{2}} \frac{\sinh ^{2}\left(c \Delta \tau \sqrt{k_{c}^{2}-k^{2}}\right)}{k_{c}^{2}-k^{2}} .
$$

Once again, either for $k_{c}=0$ or for $\Delta \tau=0, N_{k<k_{c}}$ vanishes, i.e., no tunneling ever takes place. On the other hand, for $k \rightarrow k_{c}$, Eq. (43) is reduced to the constant value (38), verifying that, the number of gravitons in the state denoted by $k_{c}$ is well-defined and finite.

In view of Eqs. (36), (38), and (43), it is now evident that, the number operator involved in the creation of gravitons along the transition of the Universe through a radiation-plus-strings stage is a continuous function of $k$, for every $0<k<\infty$.

Finally, for $k \ll k_{c}$, the expectation value of $N_{k<k_{c}}$ reads

$$
N_{k<k_{c}} \simeq \frac{k_{c}^{2}}{4 k^{2}} \sinh ^{2}\left(k_{c} c \Delta \tau\right)
$$

The apparent infrared divergence in Eq. (44) is fictitious. Indeed, these very low-frequency (very long-wavelength) waves are created way beyond the horizon [13]. As a consequence, they will enter inside the visible Universe at very late times, some of them (i.e., those with $k \approx 0$ ) not even today.

\section{THE POWER SPECTRUM OF THE CREATED GRAVITONS}

During the (late) radiation stage of the cosmic expansion, the physical frequency of a $\mathrm{GW}$ is defined as $\omega=$ $c k / R(\tau)$ and the number of states with frequencies in the interval $\omega$ and $\omega+d \omega$ is written in the form $d n_{\omega}=$ $\omega^{2} d \omega / 2 \pi^{2} c^{3}$. Accordingly, the distribution of the gravitational-energy density, summed over the two polarization states of the gravitons present in the late-radiation epoch, is given by

$$
d \epsilon_{\mathrm{gw}}=\mathcal{P}(\omega) d \omega=2 \hbar \omega \frac{\omega^{2}}{2 \pi^{2} c^{3}}\left|\beta_{k}\right|^{2} d \omega .
$$

Once again, we distinguish the following cases:

\section{A. Oscillating modes}

In terms of $k>k_{c}$, Eq. (45) is written in the form

$$
\begin{aligned}
d \epsilon_{k>k_{c}} & =\mathcal{P}\left(k>k_{c}\right) d k \\
& =\frac{1}{R^{4}(\tau)} \frac{\hbar c}{4 \pi^{2}} k_{c}^{4} \frac{\sin ^{2}\left(c \Delta \tau \sqrt{k^{2}-k_{c}^{2}}\right)}{k^{2}-k_{c}^{2}} k d k .
\end{aligned}
$$

Now, in view of Eq. (46), the corresponding power spectrum [17] reads

$$
\mathcal{P}\left(k>k_{c}\right)=\frac{d \epsilon_{k>k_{c}}}{d k}=\frac{\hbar c}{4 \pi^{2}} k_{c_{\mathrm{ph}}}^{4}(\tau) \frac{\sin ^{2}\left(c \Delta \tau \sqrt{k^{2}-k_{c}^{2}}\right)}{k^{2}-k_{c}^{2}} k,
$$

where we have introduced the (physical) critical wave number at every $\tau$, as

$$
k_{c_{\mathrm{ph}}}(\tau)=k_{c} / R(\tau) .
$$

For $k$ close to $k_{c}, \mathcal{P}\left(k>k_{c}\right) \sim k$, while, in the large- $k$ limit, it results in a damped oscillation

$$
\mathcal{P}\left(k>k_{c}\right) \sim \frac{1}{k} \sin ^{2}(k c \Delta \tau) .
$$

According to Eq. (49), the production of gravitons with $k>k_{c}$ is suppressed at high frequencies.

Similarly, the logarithmic spectrum is written in the form

$$
\begin{aligned}
\frac{d \epsilon_{k>k_{c}}}{d(\ln k)} & =k \mathcal{P}\left(k>k_{c}\right) \\
& =\frac{\hbar c}{4 \pi^{2}} k_{c_{\mathrm{ph}}}^{4}(\tau) \frac{\sin ^{2}\left(c \Delta \tau \sqrt{k^{2}-k_{c}^{2}}\right)}{k^{2}-k_{c}^{2}} k^{2} .
\end{aligned}
$$


Equation (50) becomes particularly transparent (and useful) in terms of the dimensionless parameter (see, e.g., [13])

$$
x=\frac{k}{k_{c}} \geq 1,
$$

which measures the comoving wave number of the oscillating modes in units of their ground-state counterpart, $k_{c}$. In terms of $x$, Eq. (50) is written in the form

$$
\begin{aligned}
\frac{d \epsilon_{k>k_{c}}}{d(\ln k)} & =k \mathcal{P}\left(k>k_{c}\right)=x \mathcal{P}(x>1) \\
& =\frac{\hbar c}{4 \pi^{2}} k_{c_{\mathrm{ph}}}^{4}(\tau) \frac{\sin ^{2}\left(k_{c} c \Delta \tau \sqrt{x^{2}-1}\right)}{x^{2}-1} x^{2},
\end{aligned}
$$

or, else,

$$
\begin{aligned}
x \mathcal{P}(x>1)= & 4 \pi \frac{1}{4}\left(k_{c} c \Delta \tau\right)^{2} 2 \hbar c k_{c_{\mathrm{ph}}}(\tau) \frac{1}{\lambda_{c_{\mathrm{ph}}}^{3}(\tau)} \\
& \times \frac{\sin ^{2}\left(k_{c} c \Delta \tau \sqrt{x^{2}-1}\right)}{\left(k_{c} c \Delta \tau \sqrt{x^{2}-1}\right)^{2}} x^{2},
\end{aligned}
$$

where $\lambda_{c_{\mathrm{ph}}}=2 \pi / k_{c_{\mathrm{ph}}}$ is the correlation (wave)length, associated to $k_{c_{\mathrm{ph}}}$.

At a fixed $\tau \geq \tau_{\mathrm{sc}}$, the gravitational energy contained within the volume $\lambda_{c_{\mathrm{ph}}}^{3}$, is $2 \hbar c k_{c_{\mathrm{ph}}}$, i.e., the energy of a graviton with physical wave number $k_{c_{\mathrm{ph}}}$ summed over the two polarization states. Therefore, the quantity

$$
\epsilon_{c}(\tau)=N_{k_{c}} 2 \hbar c k_{c_{\mathrm{ph}}}(\tau) \frac{1}{\lambda_{c_{\mathrm{ph}}}^{3}(\tau)}
$$

(where $N_{k_{c}}$ is given by Eq. (38)) represents the energy density of the gravitons created in the (ground) state denoted by $k_{c}$, at every $\tau \geq \tau_{\mathrm{sc}}$ and the logarithmic spectrum (53) reads

$$
x \mathcal{P}(x>1)=4 \pi \epsilon_{c}(\tau) \frac{\sin ^{2}\left(k_{c} c \Delta \tau \sqrt{x^{2}-1}\right)}{\left(k_{c} c \Delta \tau \sqrt{x^{2}-1}\right)^{2}} x^{2} .
$$

We can easily verify that, for $x=1$, one is left with the energy density of the gravitons in the state $k=k_{c}$ (within the solid angle of a sphere- $4 \pi$ ), while, as we depart from the lowest-allowed comoving wave number of the oscillating modes, the gravitational power is distributed among the various $k$-intervals according to Eq. (55). In particular, on the approach to $k_{c}$, the logarithmic spectrum is quadratic in $k$, while, for $k \gg k_{c}(x \rightarrow \infty)$, it reduces to a periodic function of the comoving wave number with constant amplitude (Fig. 1)

$$
\frac{d \epsilon_{k>k_{c}}}{d(\ln k)} \sim \sin ^{2}(k c \Delta \tau)
$$

The importance of this result rests in the fact that the logarithmic spectrum is a rescaling of the function

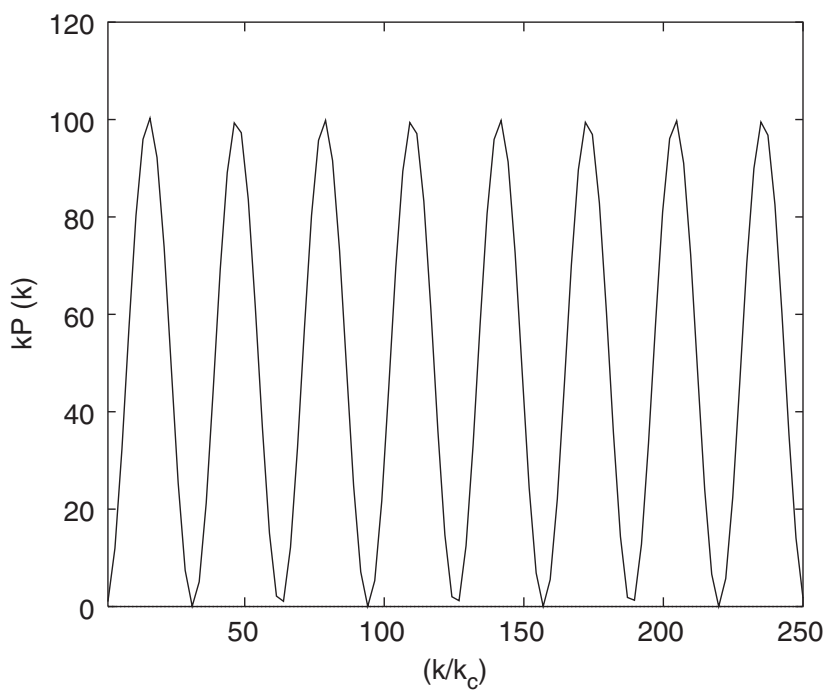

FIG. 1. The logarithmic spectrum $k \mathcal{P}(k)$, of the highfrequency part $\left(k>k_{c}\right)$ of the metric perturbations created in the scaling of a long-cosmic-string network (normalized over $4 \pi \epsilon_{c}$ ), versus $k$ in units of $k_{c}$, for $\Delta \tau=5.5 \tau_{*}$ (according to [13]).

$$
\frac{1}{\varepsilon_{\mathrm{cr}}} \frac{d \epsilon_{\mathrm{gw}}}{d(\ln k)}=\Omega_{\mathrm{gw}}=\frac{1}{\varepsilon_{\mathrm{cr}}} \frac{d \epsilon_{\mathrm{gw}}}{d(\ln f)}
$$

(where $\varepsilon_{\mathrm{cr}}$ is the critical-energy density for closing the Universe), which characterizes the intensity of a stochastic background of CGWs [36].

Therefore, if a relic CGW signal has been filtered by a long-cosmic-string network [13], its profile would have been modified along the lines of Eq. (55) [or Eq. (56)], thus resulting in a periodic function of the frequency, the period of which depends solely on the duration of the corresponding radiation-plus-strings stage (Fig. 1).

\section{B. Nonoscillating modes}

In this case, in terms of the dimensionless parameter $x=$ $\frac{k}{k_{c}}(\leq 1)$, the distribution of the gravitational-energy density of the waves with $k<k_{c}$, which are present in the lateradiation epoch, is given by

$$
d \epsilon_{k<k_{c}}=4 \pi \epsilon_{c}(\tau) \frac{\sinh ^{2}\left(k_{c} c \Delta \tau \sqrt{1-x^{2}}\right)}{\left(k_{c} c \Delta \tau \sqrt{1-x^{2}}\right)^{2}} x d x,
$$

where $\epsilon_{c}(\tau)$ is given by Eq. (54). Accordingly, the corresponding logarithmic spectrum reads (notice that, here, $\mathcal{P}_{k}$ stands for $P_{k}^{2}$ of [13])

$$
\begin{aligned}
\frac{d \epsilon_{k<k_{c}}}{d(\ln k)} & =k \mathcal{P}\left(k<k_{c}\right) \\
& =4 \pi \epsilon_{c}(\tau) \frac{\sinh ^{2}\left(k_{c} c \Delta \tau \sqrt{1-x^{2}}\right)}{\left(k_{c} c \Delta \tau \sqrt{1-x^{2}}\right)^{2}} x^{2}
\end{aligned}
$$




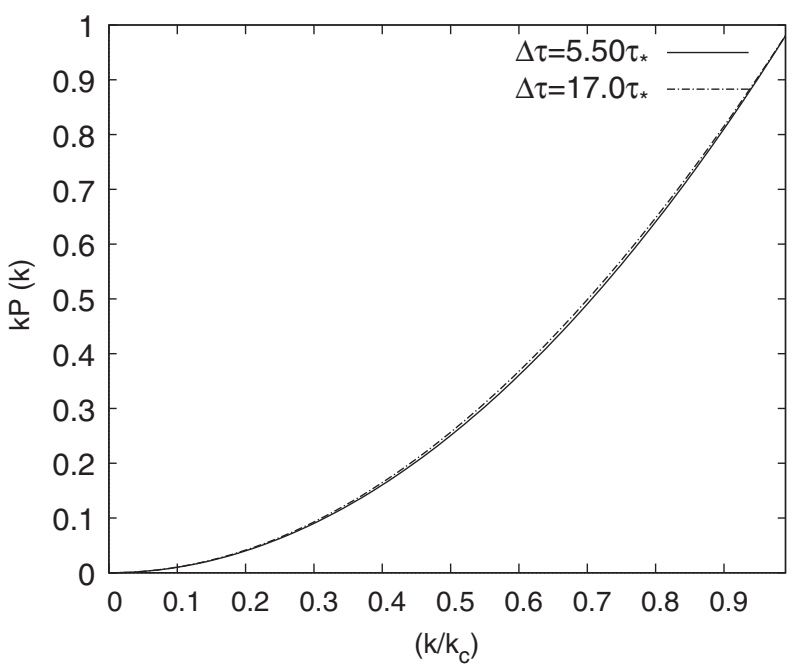

FIG. 2. The logarithmic spectrum $k \mathcal{P}(k)$, of the CGW modes with $k<k_{c}$, i.e., those created outside the horizon (normalized over $4 \pi \epsilon_{c}$ ), versus $k$ in units of $k_{c}$, for $\Delta \tau=5.5 \tau_{*}$ (solid line) and $\Delta \tau=17 \tau_{*}$ (dashed line).

and is presented in Fig. 2, which, in fact, corresponds to a (magnified) "detail" of the lower left corner of Fig. 1.

Accordingly, if the Universal evolution has gone through a radiation-plus-strings stage, then, in connection to [13], the anticipated profile of a stochastic GW signal should be modified as follows:

(i) Every GW background of cosmological origin that was created earlier than $\tau_{*}$ (e.g., the metric perturbations predicted by inflation), is being filtered by the long-cosmic-string network, thus, being discriminated to (high-frequency) oscillating modes and (low-frequency) nonoscillating ones. The separatrix between these modes is set at $k_{c}=\sqrt{\frac{8 \pi G}{3 c^{2}} M_{2}}$, where $M_{2}$ is the initial amount of the linear defects, corresponding to a present-time frequency, $f_{c}$, given by Eq. 56 of [13].

(ii) Because of quantum-gravitational effects, the highfrequency part $\left(k>k_{c}\right)$ of the corresponding CGW signal is being further amplified by a factor of 100 (see, e.g., Fig. 1), while, its profile is turned to a periodic function of the frequency, the period of which depends (solely) on the duration $(\Delta \tau)$ of the radiation-plus-strings stage. To the best of our knowledge, such an amplification mechanism of a stochastic GW signal has not yet been considered elsewhere.

(iii) According to our model, the only interference between an early-radiation epoch and the lateradiation era comes from a period of significant presence of cosmic strings (encapsulated in the cosmological model). Therefore, it is natural to assume that any energization of the gravitationalperturbation field (graviton production) is due to a corresponding energy-loss of the long-lineardefects network. In this context, it is possible that the actual scaling process might have been completed a little bit earlier than what is currently anticipated by the scientific community (see, e.g., [29,30,32]).

As we have already mentioned, the number of gravitons, $N_{k_{c}}$, created in the ground state of the oscillating metric perturbations, is well-defined and finite. These modes enter inside the horizon at some (coordinate) time $t_{c}>t_{\mathrm{sc}}$, at which, their physical wavelength (defined as $\lambda_{\mathrm{ph}}\left(t_{c}\right)=$ $\lambda_{c} R\left(t_{c}\right)$ ) becomes smaller than the corresponding Hubble radius $\left(\ell_{H}\left(t_{c}\right)=c / H\left(t_{c}\right), H\right.$ being the Hubble parameter). According to [13], we have

$$
t_{c} \geq \frac{3 \pi}{11 \gamma_{*}^{2}}\left(\frac{G \mu}{c^{2}}\right)^{-2} t_{\mathrm{cr}}
$$

where $t_{\mathrm{cr}}$ is the time at which the Universe acquires the critical temperature below which the cosmic strings are formed (for GUT-scale strings, $t_{\mathrm{cr}} \sim 10^{-31} \mathrm{sec}$ ) and $\gamma_{*}$ is the number of correlation lengths $\left(4 \leq \gamma_{*} \leq 7\right)$ inside the horizon at $t_{*}=\left(\frac{G \mu}{c^{2}}\right)^{-1} t_{\text {cr }}$ (see, e.g., [6]).

The dimensionless quantity $\left(G \mu / c^{2}\right)$ characterizes the strength of the gravitational interactions of strings. The observations give an upper limit on the value of this parameter. In particular, the current $\mathrm{CMB}$ bound is [37] $\left(G \mu / c^{2}\right) \leq 1.3 \times 10^{-6}$ while more recent studies on a cosmic-string contribution in the WMAP data [38] have yielded the tighter bound $\left(G \mu / c^{2}\right) \leq 3.3 \times 10^{-7}$, something that is also confirmed by gravitational lensing observations: $\left(G \mu / c^{2}\right) \geq 4 \times 10^{-7}[39,40]$. Therefore, in what follows, we may consider $\left(G \mu / c^{2}\right)=\frac{1}{\gamma_{*}} \times 10^{-6}$, so that, $t_{c} \gtrsim 10^{12} t_{\mathrm{cr}} \sim 10^{-19} \mathrm{sec}$.

As far as the oscillating modes are concerned, $k_{c}$ is the lowest allowed comoving wave number. Therefore, after $t_{c}$, no oscillating CGW modes (created by this mechanism) are left, to enter inside the Hubble sphere. For $t>t_{c}$, the only contribution to the CGW-energy density comes from the low-frequency, nonoscillatory modes, of comoving wave number $k<k_{c}$.

\section{THE EXTRA GW-ENERGY DENSITY}

Now, we may proceed even further, to calculate the (integrated) extra GW-energy density present in the lateradiation epoch, due to the metric perturbations created in the scaling of the long-cosmic-string network. According to what was previously stated, two kinds of modes are present in the late-radiation era. We consider each case separately:

\section{A. Oscillating modes}

The integrated energy density of the oscillating modes $\left(k>k_{c}\right)$, created in the transition of the Universe through the radiation-plus-strings stage, is given by 


$$
\mathcal{E}_{\mathrm{gw}}\left(k>k_{c}\right)=\int_{k_{c}}^{\infty} d \epsilon_{k>k_{c}}(k)=\int_{1}^{\infty} d \epsilon_{k>k_{c}}(x),
$$

which, in view of Eqs. (38) and (55), is written in the form

$$
\begin{aligned}
\mathcal{E}_{\mathrm{gw}}\left(k>k_{c}\right)= & \pi \epsilon_{c}(\tau) \frac{1}{N_{k_{c}}} \\
& \times \int_{1}^{\infty} \frac{\sin ^{2}\left(2 \sqrt{N_{k_{c}}} \sqrt{x^{2}-1}\right)}{x^{2}-1} x d x
\end{aligned}
$$

or else,

$$
\begin{aligned}
& \mathcal{E}_{\mathrm{gw}}\left(k>k_{c}\right) \\
& =4 \pi \epsilon_{c}(\tau) \frac{1}{N_{k_{c}}} \\
& \quad \times \int_{1}^{\infty} \frac{\sin ^{2}\left(\sqrt{N_{k_{c}}} \sqrt{x^{2}-1}\right) \cos ^{2}\left(\sqrt{N_{k_{c}}} \sqrt{x^{2}-1}\right)}{x^{2}-1} x d x .
\end{aligned}
$$

In fact, the high-frequency gravitons, responsible for the infinite upper limit, can be produced only by an instantaneous change in the scalar curvature. In our case, the highest frequency (which can be produced) is determined by the speed of the transition (the duration of the radiationplus-strings stage), otherwise particle creation is adiabatically suppressed (see, e.g., [20,21,34]). Hence,

$$
\gamma k c=c \sqrt{k^{2}-k_{c}^{2}} \leq \frac{1}{\Delta \tau}
$$

[cf. Equations (21) and (22)] and, therefore,

$$
\chi=\sqrt{x^{2}-1} \leq \frac{1}{2 \sqrt{N_{k_{c}}}} .
$$

Now, in terms of the parameter $\chi$, Eq. (63) is written in the form

$$
\begin{aligned}
\mathcal{E}_{\mathrm{gw}}\left(k>k_{c}\right)= & 4 \pi \epsilon_{c}(\tau) \frac{1}{N_{k_{c}}} \\
& \times \int_{0}^{\left(1 / 2 \sqrt{N_{k_{c}}}\right)} \frac{\sin ^{2}\left(\sqrt{N_{k_{c}}} \chi\right) \cos ^{2}\left(\sqrt{N_{k_{c}}} \chi\right)}{\chi} d \chi
\end{aligned}
$$

and the infinite upper limit can be recovered under the assumption $N_{k_{c}} \ll 1$ (in fact, this assumption is quite accurate, since, according to [13], $N_{k_{c}}=2 \times 10^{-3}$ ). However, in this case, we have

$$
\begin{aligned}
& \sin \left(\sqrt{N_{k_{c}}} \chi\right)=N_{k_{c}}^{1 / 2} \chi-\frac{1}{6} N_{k_{c}}^{3 / 2} \chi^{3}+O\left(N_{k_{c}}^{5 / 2}\right) \\
& \cos \left(\sqrt{N_{k_{c}}} \chi\right)=1-\frac{1}{2} N_{k_{c}} \chi^{2}+O\left(N_{k_{c}}^{2}\right)
\end{aligned}
$$

Accordingly, discarding terms of order $O\left(N_{k_{c}}^{2}\right)$, Eq. (66) is written in the form

$$
\mathcal{E}_{\mathrm{gw}}\left(k>k_{c}\right) \simeq 4 \pi \epsilon_{c}(\tau) \int_{0}^{\left(1 / 2 \sqrt{N_{k_{c}}}\right)}\left(\chi-\frac{4}{3} N_{k_{c}} \chi^{3}\right) d \chi,
$$

thus resulting in

$$
\mathcal{E}_{\mathrm{gw}}\left(k>k_{c}\right) \simeq \frac{5 \pi}{12} \epsilon_{c}(\tau) \frac{1}{N_{k_{c}}} .
$$

By virtue of Eq. (54) and (69) takes on its final form

$$
\mathcal{E}_{\mathrm{gw}}\left(k>k_{c}\right) \simeq \frac{1}{R^{4}(t)} \frac{5 \hbar c}{48 \pi^{2}} k_{c}^{4} .
$$

Inside the Hubble sphere of the late-radiation era, the energy density of the gravitons with $k>k_{c}$ which were created at earlier times $t_{\mathrm{sc}} \leq t^{\prime}<t$ and the wavelength of which became less than the horizon due to the expansion of the Universe at $t_{\mathrm{sc}} \leq t \leq t_{c}$, is given by the sum of all the previous increments $\delta \mathcal{E}_{\mathrm{gw}}\left(t^{\prime}\right)$ being redshifted by the factor $R^{4}\left(t^{\prime}\right) / R^{4}(t)$

$$
\begin{aligned}
& \varepsilon_{\mathrm{gw}}\left(t_{\mathrm{sc}} \leq t \leq t_{c}\right) \\
& =\int_{t_{\mathrm{sc}}}^{t_{c}} \frac{R^{4}\left(t^{\prime}\right)}{R^{4}(t)}\left(-\frac{d}{d t^{\prime}} \mathcal{E}_{\mathrm{gw}}\right) d t^{\prime} \Rightarrow \varepsilon_{\mathrm{gw}}\left(t_{\mathrm{sc}} \leq t \leq t_{c}\right) \\
& =\frac{5 \hbar c}{24 \pi^{2}} k_{\mathrm{ph}_{\mathrm{ph}}}^{4}\left(t_{\mathrm{sc}}\right)\left(\frac{t_{\mathrm{sc}}}{t_{c}}\right)^{2} \ln \left(\frac{t_{c}}{t_{\mathrm{sc}}}\right) .
\end{aligned}
$$

For $t>t_{c}$ (i.e., deep inside the late-radiation era), this constant amount of energy density is being (further) redshifted due to the cosmological expansion. Therefore,

$$
\begin{aligned}
\varepsilon_{\mathrm{gw}}^{k>k_{c}}(t) & =\varepsilon_{\mathrm{gw}}\left(t_{\mathrm{sc}} \leq t \leq t_{c}\right) \frac{R^{4}\left(t_{c}\right)}{R^{4}(t)} \Rightarrow \varepsilon_{\mathrm{gW}}^{k>k_{c}}(t) \\
& =\frac{5 \hbar c}{24 \pi^{2}} k_{\mathrm{ph}^{4}}^{4}\left(t_{c}\right)\left(\frac{t_{c}}{t}\right)^{2} \ln \left(\frac{t_{c}}{t_{\mathrm{sc}}}\right),
\end{aligned}
$$

while, for $t \geq t_{c}$, there are only the nonoscillatory modes available to enter inside the horizon and contribute to the extra GW-energy density.

\section{B. Nonoscillating modes}

The extra GW-energy density (present in the lateradiation era) of the modes with $k<k_{c}$, created in the transition of the Universe through the radiation-plusstrings stage, is given by

$$
\mathcal{E}_{\mathrm{gw}}\left(k<k_{c}\right)=\int_{0}^{k_{c}} d \epsilon_{k<k_{c}}(k)=\int_{0}^{1} d \epsilon_{k<k_{c}}(x),
$$

which, by virtue of Eqs. (38) and (58), is written in the form 


$$
\begin{aligned}
\mathcal{E}_{\mathrm{gw}}\left(k<k_{c}\right)= & \pi \epsilon_{c}(\tau) \frac{1}{N_{k_{c}}} \\
& \times \int_{0}^{1} \frac{\sinh ^{2}\left(2 \sqrt{N_{k_{c}}} \sqrt{1-x^{2}}\right)}{1-x^{2}} x d x .
\end{aligned}
$$

Now, in terms of the parameter $\phi=\sqrt{1-x^{2}}$, Eq. (74) reads

$$
\begin{aligned}
\mathcal{E}_{\mathrm{gw}}\left(k<k_{c}\right)= & 4 \pi \epsilon_{c}(\tau) \frac{1}{N_{k_{c}}} \\
& \times \int_{0}^{1} \frac{\sinh ^{2}\left(\sqrt{N_{k_{c}}} \phi\right) \cosh ^{2}\left(\sqrt{N_{k_{c}}} \phi\right)}{\phi} d \phi,
\end{aligned}
$$

which, in the limit $N_{k_{c}} \ll 1$, results in

$$
\mathcal{E}_{\mathrm{gw}}\left(k<k_{c}\right) \simeq 4 \pi \epsilon_{c}(\tau) \int_{0}^{1}\left(\phi+\frac{4}{3} N_{k_{c}} \phi^{3}\right) d \phi,
$$

yielding

$$
\mathcal{E}_{\mathrm{gw}}\left(k<k_{c}\right) \simeq 2 \pi \epsilon_{c}(\tau)\left(1+\frac{2}{3} N_{k_{c}}\right),
$$

or else,

$$
\mathcal{E}_{\mathrm{gw}}\left(k<k_{c}\right) \simeq \frac{1}{R^{4}(t)} N_{k_{c}} \frac{\hbar c}{2 \pi^{2}} k_{c}^{4} .
$$

For the same reasons that led to Eq. (71), the energy density of the gravitons with $k<k_{c}$ which were created at earlier times $t_{c} \leq t^{\prime}<t$ and the wavelength of which became less than the horizon due to the expansion of the Universe at $t_{c} \leq t \leq t_{\text {rec }}$ (where $t_{\text {rec }}$ is the recombination time), is given by

$$
\varepsilon_{\mathrm{gw}}^{k<k_{c}}(t)=N_{k_{c}} \frac{\hbar c}{\pi^{2}} k_{\mathrm{phh}^{4}}^{4}\left(t_{c}\right)\left(\frac{t_{c}}{t}\right)^{2} \ln \left(\frac{t}{t_{c}}\right)
$$

\section{The overall energy density}

With the aid of Eqs. (72) and (79), we can determine the (overall) extra GW-energy density, due to the metric perturbations created in the scaling of a long-cosmic-string network and entered inside the horizon at $t_{c} \leq t \leq t_{\mathrm{rec}}$. We obtain

$$
\begin{aligned}
\varepsilon_{\mathrm{gw}}^{\mathrm{rps}}\left(t \geq t_{c}\right)= & \frac{\hbar c}{\pi^{2}} k_{c_{\mathrm{ph}}}^{4}\left(t_{c}\right)\left(\frac{t_{c}}{t}\right)^{2} \\
& \times[\underbrace{\frac{5}{24} \ln \left(\frac{t_{c}}{t_{\mathrm{sc}}}\right)}_{\text {oscillating }}+\underbrace{N_{k_{c}} \ln \left(\frac{t}{t_{c}}\right)}_{\text {non-oscillating }}],
\end{aligned}
$$

where we have marked the contribution of both the oscillating and the nonoscillating modes. By definition, the physical wavelength of the mode $k_{c}$ at $t_{c}$ equals the horizon length (at $t_{c}$ this mode enters into the visible Universe), so that

$$
k_{c_{\mathrm{ph}}}\left(t_{c}\right)=\frac{2 \pi}{\lambda_{c_{\mathrm{ph}}}\left(t_{c}\right)}=\frac{2 \pi}{\ell_{H}\left(t_{c}\right)}=\frac{2 \pi H\left(t_{c}\right)}{c} .
$$

Accordingly, Eq. (80) is written in the form

$$
\begin{aligned}
\varepsilon_{\mathrm{gw}}^{\mathrm{rps}}\left(t \geq t_{c}\right)= & 16 \pi^{2} \frac{\hbar}{c^{3}} H^{4}\left(t_{c}\right)\left(\frac{t_{c}}{t}\right)^{2} \\
& \times\left[\frac{5}{24} \ln \left(\frac{t_{c}}{t_{\mathrm{sc}}}\right)+N_{k_{c}} \ln \left(\frac{t}{t_{c}}\right)\right],
\end{aligned}
$$

which, in view of the Appendix A, results in

$$
\begin{aligned}
\frac{\varepsilon_{\mathrm{gw}}^{\mathrm{rps}}}{\varepsilon_{\mathrm{gW}}^{\mathrm{inf}}}\left(t \geq t_{c}\right)= & \frac{247808}{81} \pi^{2} \gamma_{*}^{4}\left(\frac{G \mu}{c^{2}}\right)^{4}\left[\frac{H_{\mathrm{cr}}}{H_{\mathrm{inf}}}\right]^{2}\left(\frac{t_{0}}{t}\right)^{2} \\
& \times\left[\frac{5}{24} \ln \left(\frac{t_{c}}{t_{\mathrm{sc}}}\right)+N_{k_{c}} \ln \left(\frac{t}{t_{c}}\right)\right],
\end{aligned}
$$

where $\varepsilon_{\mathrm{gw}}^{\mathrm{inf}}$ is the GW-energy density predicted by (de Sitter-type) inflation, $H_{\text {inf }}$ and $H_{\text {cr }}$ are the values of the Hubble parameter at inflation and at $t_{\mathrm{cr}}$, respectively, while $t_{0} \simeq 4.3 \times 10^{17} \mathrm{sec}$ denotes the present epoch. According to Allen [20], during the inflationary epoch, $H_{\text {inf }}=8 \times 10^{34} \mathrm{sec}^{-1}$, while, within the late-radiation era, $H_{\mathrm{cr}}=\left(2 t_{\mathrm{cr}}\right)^{-1}=5 \times 10^{30} \mathrm{sec}^{-1}$. Finally, admitting that $\Delta \tau=5.5 \tau_{*}$ (see, e.g., [13]), we obtain $N_{k_{c}}=$ $2 \times 10^{-3}, t_{\mathrm{sc}} \simeq 30 t_{*}$ and, therefore, $t_{c} \simeq 10^{4} t_{\mathrm{sc}}$. Inserting these values into Eq. (83) we obtain

$$
\frac{\varepsilon_{\mathrm{gw}}^{\mathrm{rps}}}{\varepsilon_{\mathrm{gw}}^{\mathrm{iff}}}\left(t \geq t_{c}\right)=4.28 \times 10^{7}\left(\frac{\mathrm{sec}}{t}\right)^{2}\left[1+10^{-3} \ln \left(\frac{t}{\mathrm{sec}}\right)\right] .
$$

We observe that, at late times, the only contribution to $\varepsilon_{\mathrm{gw}}^{\mathrm{rps}}$ comes from the second term in the brackets on the righthand side of Eq. (84), i.e., from the (low-frequency) nonoscillatory CGW modes (a not unexpected result). Nevertheless, within the late-radiation era, this term introduces only minor corrections to the $\frac{1}{t^{2}}$ behavior of the CGW-energy density.

It is generally admitted that the main source of relic gravitational radiation is the inflationary amplification of metric perturbations, taken place as early as $10^{-34} \mathrm{sec}$ after the big bang (see, e.g., [11]). Quite later, 
$t \geq t_{\mathrm{sc}} \sim 10^{-23} \mathrm{sec}$, after being filtered by a long-cosmicstring network, the original number of the relic (inflationary) GWs is further increased by an additional amount of CGWs [cf. Equations (36) and (43)], due to the quantumgravitational creation of gravitons along the transition of the Universe through the radiation-plus-strings stage. In this case, Eq. (84) indicates something very interesting: At times $t_{c} \sim 10^{-19} \mathrm{sec} \leq t \leq 6540 \mathrm{sec}$, the contribution of these gravitons, $\varepsilon_{\mathrm{gw}}^{\mathrm{rps}}$, to the (already) existing gravitationalenergy density $\varepsilon_{\mathrm{gw}}^{\mathrm{inf}}$, is (much) larger than $\varepsilon_{\mathrm{gw}}^{\mathrm{inf}}$ itself.

Certainly, at late times, the amount, $\varepsilon_{\mathrm{gw}}^{\mathrm{rps}}$, of the extra gravitational-energy density (created in the scaling of a long-cosmic-string network) becomes negligible, as a consequence of the cosmological expansion. Nevertheless, it may have affected significantly the cosmological evolution within the first (and most interesting) $109 \mathrm{~min}$ of the history of the Universe, thus leaving imprints on the anticipated stochastic GW power spectrum. Accordingly, for $f \geq f_{c}$ (see, e.g., [13]), the spectral intensity $\Omega_{\mathrm{gw}}$ of the CGW signal is turned to a periodic function of the frequency, while being amplified by (almost) 2 orders of magnitude (Fig. 1).

\section{CONCLUSIONS}

Assuming that there was a period in the early Universe in which the matter content could be modeled by a twocomponent fluid (consisting of radiation and a cosmicstring fluid), we demonstrate that the existence of such a (radiation-plus-strings) stage would have resulted in a measurable effect on the CGWs' power spectrum. In fact, along the transition from an early-radiation epoch to the late-radiation era through the radiation-plus-strings stage, gravitons with a highly recognizable profile could have been produced.

As a consequence, at high frequencies, the CGW spectrum would no longer be scale-invariant, but, rather, a periodic function of the frequency, the period of which depends (solely) on the duration of the radiation-plusstrings stage.

But, what is more important for the detection of CGWs, is that, the extra amount of metric perturbations created in the scaling of a long-cosmic-string network would lead to the amplification of the relic $G W$ signal by (almost) 2 orders of magnitude.

The extra-energy density of the CGWs created in the transition from an early-radiation epoch to the lateradiation era through the radiation-plus-strings stage, not only is added to the corresponding inflationary quantity. In fact, at times $10^{-19} \mathrm{sec} \leq t \leq 6540 \mathrm{sec}$, i.e., within the first (and, certainly, most interesting) $109 \mathrm{~min}$ of the Universal evolution, dominates over every other form of GW-energy density.

According to our model, the only interference between an early-radiation epoch and the late-radiation era comes from a period of a significant presence of cosmic strings (encapsulated in the cosmological model). Therefore, it is natural to assume that any energization of the gravitational-perturbation field (graviton production) is due to a corresponding energy loss of the long-lineardefects network. In this article we have neglected the backreaction of the created gravitons on the curved cosmological background (it will be the scope of a future work); nevertheless, we expect that the actual scaling process might have been completed a little bit earlier than what is currently anticipated by the scientific community.

\section{ACKNOWLEDGMENTS}

The first author (K. K.) would like to thank the Research Committee of the Technological Education Institute of Serres, for the financial support under the grant SAT/ME/ 210911-100/03, and Enric Verdaguer acknowledges support by the Spanish Research Projects MEC FPA-200766665, CPAN CSD-2007-00042, and AGAUR 2009-SGR00168 .

\section{APPENDIX A}

The contribution of the gravitons created in the transition from an early-radiation epoch to the late-radiation era through a radiation-plus-strings stage to the total amount of the gravitational-energy density in the Universe, is given by

$$
\begin{aligned}
\varepsilon_{\mathrm{gW}}^{\mathrm{rps}}\left(t \geq t_{c}\right)= & 16 \pi^{2} \frac{\hbar}{c^{3}} H^{4}\left(t_{c}\right)\left(\frac{t_{c}}{t}\right)^{2} \\
& \times\left[\frac{5}{24} \ln \left(\frac{t_{c}}{t_{\mathrm{sc}}}\right)+N_{k_{c}} \ln \left(\frac{t}{t_{c}}\right)\right]
\end{aligned}
$$

[cf. Equation (82)], which, upon consideration of the Friedmann equation

$$
H^{2}\left(t_{c}\right)=\frac{8 \pi G}{3 c^{2}} \varepsilon_{\mathrm{rad}}\left(t_{c}\right),
$$

reads

$$
\begin{aligned}
\varepsilon_{\mathrm{gW}}^{\mathrm{rps}}\left(t \geq t_{c}\right)= & \frac{128 \pi^{3}}{3} H^{2}\left(t_{c}\right) t_{\mathrm{Pl}}^{2} \varepsilon_{\mathrm{rad}}(t) \\
& \times\left[\frac{5}{24} \ln \left(\frac{t_{c}}{t_{\mathrm{sc}}}\right)+N_{k_{c}} \ln \left(\frac{t}{t_{c}}\right)\right],
\end{aligned}
$$

where $t_{\mathrm{Pl}}=5.44 \times 10^{-44} \mathrm{sec}$ is the Planck time. Taking into account that, in terms of $\varepsilon_{\text {rad }}\left(t_{c}\right)$, the mass-energy density in the matter-dominated era is given by

$$
\varepsilon_{\text {matt }}(t)=\varepsilon_{\text {rad }}\left(t_{c}\right)\left(\frac{t_{c}}{t_{0}}\right)^{2},
$$

where $t_{0}$ denotes the present epoch, we find that 


$$
\begin{aligned}
\varepsilon_{\mathrm{gw}}^{\mathrm{rps}}\left(t \geq t_{c}\right)= & \frac{128 \pi^{3}}{3} H^{2}\left(t_{c}\right) t_{\mathrm{Pl}}^{2} \varepsilon_{\text {matt }}(t)\left(\frac{t_{0}}{t}\right)^{2} \\
& \times\left[\frac{5}{24} \ln \left(\frac{t_{c}}{t_{\mathrm{sc}}}\right)+N_{k_{c}} \ln \left(\frac{t}{t_{c}}\right)\right],
\end{aligned}
$$

which, upon definition of $H_{\text {inf }}$ as the Hubble parameter at (de Sitter) inflation, can be written in the more convenient form

$$
\begin{aligned}
\varepsilon_{\mathrm{gw}}^{\mathrm{rps}}\left(t \geq t_{c}\right)= & \frac{3}{16 \pi} H_{\mathrm{inf}}^{2} t_{\mathrm{Pl}}^{2} \frac{2048}{9} \pi^{4}\left[\frac{H\left(t_{c}\right)}{H_{\mathrm{inf}}}\right]^{2} \varepsilon_{\mathrm{matt}}(t)\left(\frac{t_{0}}{t}\right)^{2} \\
& \times\left[\frac{5}{24} \ln \left(\frac{t_{c}}{t_{\mathrm{sc}}}\right)+N_{k_{c}} \ln \left(\frac{t}{t_{c}}\right)\right]
\end{aligned}
$$

and, by virtue of Eq. (4.10) of [20] (or Eq. 16 of [34]), takes on its final form

$$
\begin{aligned}
\varepsilon_{\mathrm{gw}}^{\mathrm{rps}}\left(t \geq t_{c}\right)= & \varepsilon_{\mathrm{gw}}^{\inf } \frac{2048}{9} \pi^{4}\left[\frac{H\left(t_{c}\right)}{H_{\mathrm{inf}}}\right]^{2}\left(\frac{t_{0}}{t}\right)^{2} \\
& \times\left[\frac{5}{24} \ln \left(\frac{t_{c}}{t_{\mathrm{sc}}}\right)+N_{k_{c}} \ln \left(\frac{t}{t_{c}}\right)\right] .
\end{aligned}
$$

Eventually, taking into account that, in a FRW radiation model we have $\frac{H\left(t_{c}\right)}{H_{\mathrm{cr}}}=\frac{t_{\mathrm{cr}}}{t_{c}}$, with the aid of Eq. (60) and (A7), results in Eq. (83).
[1] S. Weinberg, Gravitation and Cosmology (Wiley, New York, 1972).

[2] M. Maggiore, Phys. Rep. 331, 283 (2000).

[3] C. W. Misner, K. S. Thorne, and J. A. Wheeler, Gravitation (Freeman, San Francisco, 1973).

[4] L. P. Grishchuk and A. G. Polnarev, in General Relativity and Gravitation-One Hundred Years After the Birth of Albert Einstein, edited by A. Held (Plenum, New York, 1980).

[5] T.B.W. Kibble, Nucl. Phys. B252, 227 (1985).

[6] M. B. Hindmarsh and T. B. W. Kibble, Rep. Prog. Phys. 58, 477 (1995).

[7] A. Vilenkin and E.P.S. Shellard, Cosmic Strings and Other Topological Defects (Cambridge University Press, Cambridge, England, 2000).

[8] A. Vilenkin, Nature (London) 322, 613 (1986).

[9] A. Vilenkin, Phys. Rev. Lett. 46, 1169 (1981).

[10] Ya. B. Zel' dovich, Mon. Not. R. Astron. Soc. 192, 663 (1980).

[11] B. Allen, in The Stochastic Gravity-Wave Background: Sources and detection, edited by J. A. Marck and J.P. Lassota, Les Houches School on Astrophysical Sources of Gravitational Waves (Cambridge University Press, Cambridge, 1997).

[12] R. A. Battye, R. R. Caldwell, and E.P.S. Shellard, in Topological Defects in Cosmology, edited by $\mathrm{F}$. Melchiorri and M. Signore (World Scientific, Singapore, 1998).

[13] K. Kleidis, D. B. Papadopoulos, E. Verdaguer, and L. Vlahos, Phys. Rev. D 78, 024027 (2008).

[14] L.P. Grishchuk, Sov. Phys. JETP 40, 409 (1975).

[15] L. Ford and L. Parker, Phys. Rev. D 16, 245 (1977).

[16] L. Ford and L. Parker, Phys. Rev. D 16, 1601 (1977).

[17] V. F. Mukhanov, H. A. Feldman, and R. H. Brandenberger, Phys. Rep. 215, 203 (1992).
[18] U. Bleyer, D. E. Liebscher, and A. G. Polnarev, Classical Quantum Gravity 8, 477 (1991).

[19] A. Vilenkin, Phys. Rev. D 24, 2082 (1981).

[20] B. Allen, Phys. Rev. D 37, 2078 (1988).

[21] N.D. Birrell and P.C.W. Davies, Quantum Fields in Curved Space (Cambridge University Press, Cambridge, England, 1982).

[22] T. B. W. Kibble, G. Lazarides, and Q. Shafi, Phys. Rev. D 26, 435 (1982).

[23] E. W. Kolb and M.S. Turner, The Early Universe (Addison-Wesley, New York, 1990).

[24] T.B.W. Kibble, J. Phys. A 9, 1387 (1976).

[25] T. B. W. Kibble, in Cosmology and Particle Physics, edited by E. Alvarez, R. Dominguez-Tenreiro, J. M. IbanezCabanel, and M. Quiros, Lectures at GIFT XVIIth International Seminar on Theoretical Physics (World Scientific, Singapore, 1987).

[26] A. Vilenkin, Phys. Rev. D 43, 1060 (1991).

[27] A. Albrecht and N. Turok, Phys. Rev. D 40, 973 (1989).

[28] D. P. Bennett and F. R. Bouchet, Phys. Rev. Lett. 63, 2776 (1989).

[29] D. P. Bennett and F. R. Bouchet, Phys. Rev. D 41, 2408 (1990).

[30] B. Allen B and E. P. S. Shellard, Phys. Rev. Lett. 64, 119 (1990).

[31] M. Sakellariadou, J. Cosmol. Astropart. Phys. 04 (2005) 003.

[32] C. Ringeval, M. Sakellariadou, and F. R. Bouchet, J. Cosmol. Astropart. Phys. 02 (2007) 023.

[33] C. J. A. P. Martins and E. P. S. Shellard, Phys. Rev. D 73, 043515 (2006).

[34] V. Sahni, Phys. Rev. D 42, 453 (1990).

[35] L. I. Schiff, Quantum Mechanics (Mc Graw-Hill, New York, 1955).

[36] B. J. Carr, Astron. Astrophys. 89, 6 (1980). 
[37] L. Pogosian, S-H. H. Tye, I. Wasserman, and M. Wyman, Phys. Rev. D 68, 023506 (2003).

[38] E. Jeong and G. F. Smoot, arXiv:astro-ph/0406432.

[39] M. Sazhin, G. Longo, M. Capaccioli, J. M. Alcala, R. Silvotti, G. Covone, O. Khovanskaya, M. V. Pavlov, M.
Pannella, M. Radovich, and V. Testa, Mon. Not. R. Astron. Soc. 343, 353 (2003).

[40] M. Sazhin, O. Khovanskaya, M. Capaccioli, G. Longo, J. M. Alcala, R. Silvotti, and M. V. Pavlov, arXiv:astro-ph/ 0406516. 